\title{
Peripartum Cardiomyopathy Presenting with Syncope due to Torsades de Pointes: a Case of Long QT Syndrome with a Novel KCNH2 Mutation
}

\author{
Orie Nishimoto $^{1}$, Morihiro Matsuda ${ }^{1,3}$, Kei Nakamoto ${ }^{1}$, Hirohiko Nishiyama ${ }^{1}$, \\ Kazuya Kuraoka ${ }^{2}$, Kiyomi Taniyama ${ }^{2,3}$, Ritsu Tamura ${ }^{1}$, \\ Wataru Shimizu ${ }^{4}$ and Toshiharu Kawamoto ${ }^{1}$
}

\begin{abstract}
Peripartum cardiomyopathy (PPCM) is a cardiomyopathy of unknown cause that occurs in the peripartum period. We report a case of PPCM presenting with syncope 1 month after an uncomplicated delivery. Electrocardiography showed Torsades de pointes (TdP) and QT interval prolongation. Echocardiography showed left ventricular systolic dysfunction and endomyocardial biopsy showed myocyte degeneration and fibrosis. Administration of magnesium sulfate and temporary pacing eliminated recurrent TdP. Genetic analyses revealed that recurrent TdP occurred via electrolyte disturbance and cardiac failure due to PPCM on the basis of a novel mutation in $\mathrm{KCNH} 2$, a gene responsible for inherited type 2 long QT syndrome.
\end{abstract}

Key words: Torsades de pointes, long QT syndrome, peripartum cardiomyopathy

(Intern Med 51: 461-464, 2012)

(DOI: 10.2169/internalmedicine.51.5943)

\section{Introduction}

Peripartum cardiomyopathy (PPCM) is a disease of unknown cause that occurs from 1 month antepartum to 5 months postpartum in women without preexisting heart disease (1). In most cases, PPCM presents with signs of congestive heart failure caused by severe left ventricular (LV) systolic dysfunction. A few reported cases of PPCM have featured monomorphic ventricular tachycardia (VT) (2), but no cases have shown polymorphic VT.

Torsades de pointes (TdP), a polymorphic ventricular tachycardia associated with QT interval prolongation, is a life-threatening arrhythmia that can degenerate to fatal ventricular fibrillation. Acquired QT interval prolongation can occur upon exposure to environmental stressors such as particular classes of drugs, electrolyte disturbance, heart block, or heart failure (3). Moreover, in patients with drug-induced acquired long QT syndrome (LQTS), mutations have been identified in genes encoding cardiac ion channels, such as KCNQ1, KCNH2, and SCN5A, which have proven to be associated with congenital LQTS. Here, we report a rare case of PPCM presenting with recurrent syncope due to TdP resulting from a $\mathrm{KCNH} 2$ mutation.

\section{Case Report}

A 33-year-old woman was admitted to our hospital with repeated syncope and seizures 1 month after an uncomplicated delivery. She did not have a history of pregnancyassociated diseases, spontaneous abortion or epilepsy or any symptoms of infectious disease or heart failure during pregnancy or after delivery. Prior to this pregnancy she had a delivery without any complications, and had no family history

\footnotetext{
${ }^{1}$ Department of Cardiology, National Hospital Organization, Kure Medical Center and Chugoku Cancer Center, Japan, ${ }^{2}$ Department of Diagnostic Pathology, National Hospital Organization, Kure Medical Center and Chugoku Cancer Center, Japan, ${ }^{3}$ Institute for Clinical Research, National Hospital Organization, Kure Medical Center and Chugoku Cancer Center, Japan and ${ }^{4}$ Division of Arrhythmia and Electrophysiology, Department of Cardiovascular Medicine, National Cerebral and Cardiovascular Center, Japan

Received for publication June 2, 2011; Accepted for publication September 11, 2011
}

Correspondence to Dr. Morihiro Matsuda, morihiro-m@kure-nh.go.jp 


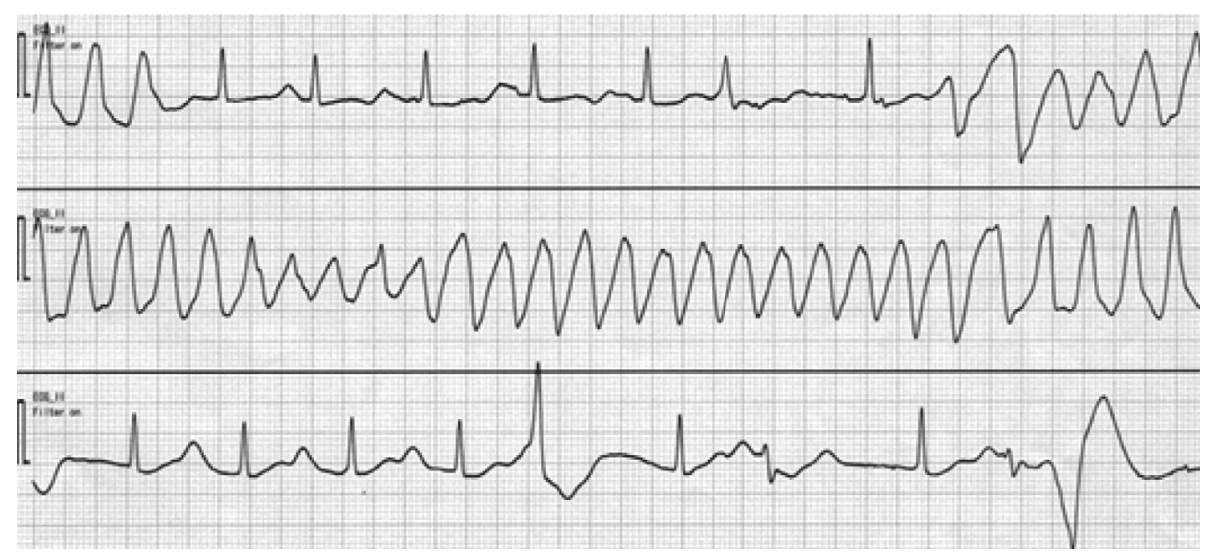

Figure 1. Electrocardiogram obtained at the time of syncope and seizure.

A

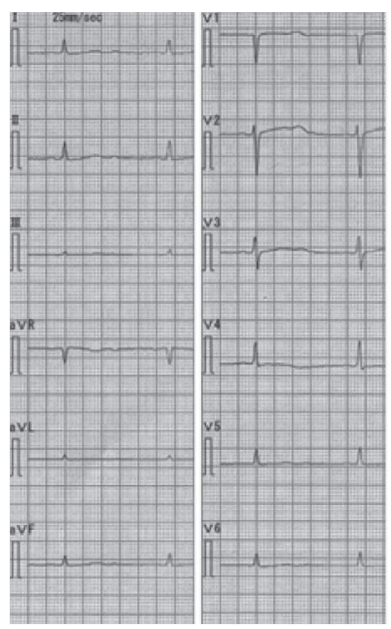

B

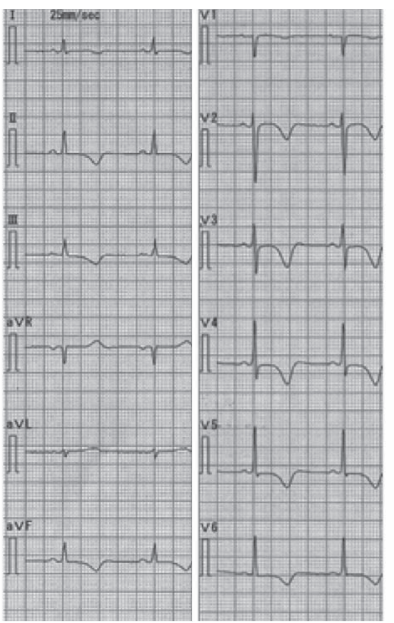

C

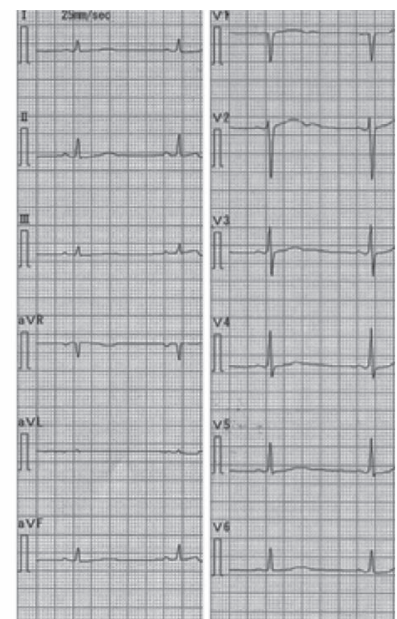

D

\begin{tabular}{lccc}
\hline & Day 1 & Day 8 & Day 120 \\
\hline QTc (ms) & 574 & 516 & 454 \\
Blood Pressure (mmHg) & $134 / 83$ & $109 / 75$ & $99 / 70$ \\
Heart Rate (bpm) & 52 & 64 & 54 \\
$\mathrm{~K}(\mathrm{mEq} / \mathrm{L})$ & 3.4 & 4.4 & 4.2 \\
$\mathrm{Mg}(\mathrm{mEq} / \mathrm{L})$ & 1.8 & $\mathrm{ND}$ & $\mathrm{ND}$ \\
\hline
\end{tabular}

ND; not determined.

Figure 2. Changes in electrocardiographic findings. Electrocardiograms obtained on admission (A), at day 8 (B), and at day 120 (C). QTc, blood pressure, heart rate, and K and Mg levels on the indicated day (D).

of heart disease or sudden death. Syncope and seizures had occurred a day before admission and the next morning and evening prior to admission, and then she was taken to the emergency room in our hospital. She had taken no medicine before admission.

The patient was alert upon admission, and the following vital signs were noted: blood pressure, $134 / 83 \mathrm{mmHg}$; pulse rate, $50 \mathrm{bpm}$; and body temperature, $36.7^{\circ} \mathrm{C}$. The patient's height and weight were $158 \mathrm{~cm}$ and $50 \mathrm{~kg}$, respectively. Physical examination did not reveal any signs of heart failure or abnormal neurological findings. Electrocardiography (ECG) showed sinus bradycardia (50 bpm) and marked QT prolongation (QTc $574 \mathrm{~ms}$ ) (Fig. 1, 2A). The chest X-ray image revealed a normal cardio-thoracic rate $(48 \%)$ without pulmonary congestion or pleural effusion. Echocardiography showed impaired LV motion with mild hypokinesis at the base and severe hypokinesis at the apex. Laboratory data showed an elevated level of white blood cells $(15,600 / \mu \mathrm{L})$, mild elevation in the levels of brain natriuretic peptide (BNP) (123.1 pg/mL), hypokalemia (K levels, $3.4 \mathrm{mEq} / \mathrm{L}$ ), and hypomagnesemia ( $\mathrm{Mg}$ levels, $1.8 \mathrm{mEq} / \mathrm{L})$, and normal serum levels of transaminases and creatinine. The levels of autoantibodies and viral antibodies were not significantly elevated, and the brain computed tomography image did not 


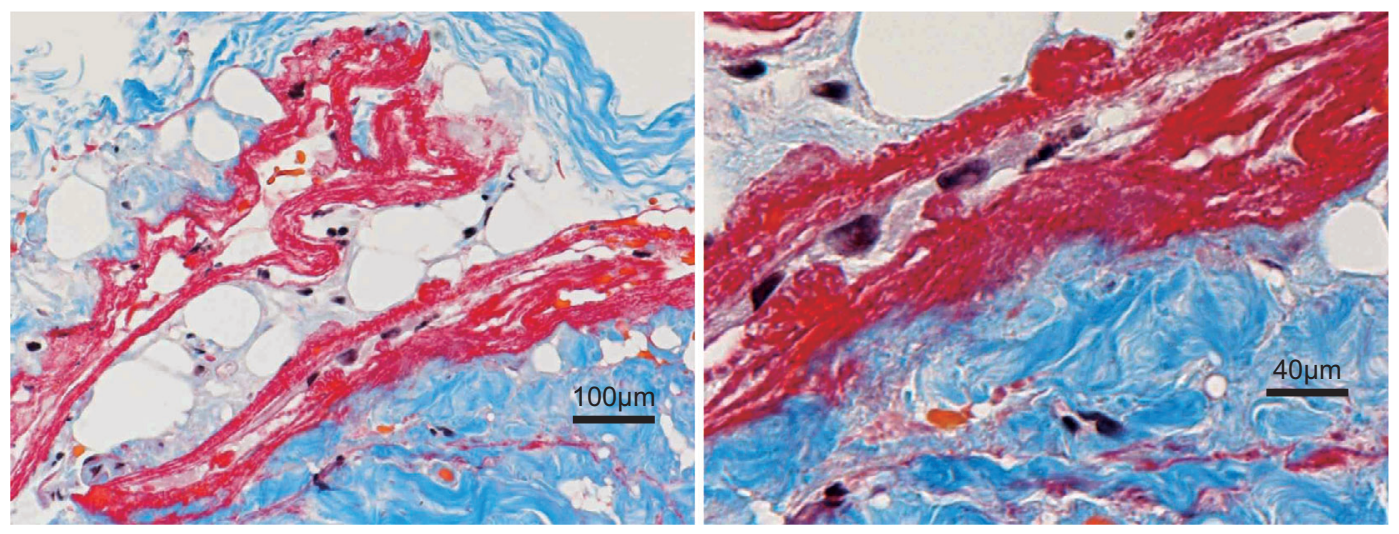

Figure 3. Endomyocardial biopsy revealing cardiomyopathy. Masson's trichrome stain.

indicate any abnormalities.

She presented with syncope and seizures immediately after admission, and the ECG monitor indicated TdP (Fig. 1) that spontaneously ceased in 10-20 s. In spite of intravenous administration of magnesium sulfate $(166 \mathrm{mEq})$, TdP recurred twice within a few hours. Correction of hypokalemia and temporary pacing at a rate of $80 \mathrm{bpm}$ effectively inhibited the repeated syncope caused by TdP. She was administered $2.5 \mathrm{mg}$ /day of enalapril after admission. After day 11, TdP did not recur even after discontinuation of temporary pacing. The patient was discharged on day 23 .

On day 3, left ventriculography showed mildly impaired LV wall motion with an ejection fraction of $43.6 \%$, although coronary angiography revealed no significant stenosis in the coronary arteries. Endomyocardial biopsy of the right ventricle revealed myocytes degeneration and interstitial fibrosis (Fig. 3). Follow-up ECGs showed that the positive or flat $\mathrm{T}$ wave in the leads I, II, III, aVF, and V2-6 was inverted until day 8 , and then gradually returned to the up-right or flat form on day 120 (Fig. 2A-C). Prolonged QTc was shortened to $516 \mathrm{~ms}$ on day 8 and $454 \mathrm{~ms}$ on day 120 (Fig. 2D). Follow-up echocardiography on day 120 showed that LV contraction was almost normal.

Genetic analyses of KCNQ1, KCNH2, and SCN5A in this patient revealed a novel mutation in the $\mathrm{KCNH} 2$ gene. This mutation involved an insertion of AGGC at exon 11 (c.2680_2681), leading to a frameshift from R894, which results in a C-terminal truncating mutation (p.R894fsX920) in $\mathrm{KCNH} 2$. This mutation was also identified in her mother and sister, resulting in border range QTc (mother, $467 \mathrm{~ms}$; and sister, $461 \mathrm{~ms}$ ) without any episodes of syncope. Her mother had experienced three uncomplicated deliveries and one spontaneous abortion, and her sister had not experienced pregnancy.

\section{Discussion}

PPCM is relatively rare, with a currently accepted estimate of an incidence of approximately 1 per 3,000 to 1 per 4,000 live births in the United States, but it can be lifethreatening, with mortality rates between $18 \%$ and $56 \%$ (1).
The present patient met the following 4 criteria for the diagnosis of PPCM, which was established by Demakis and Rahimtoola and others: 1) development of cardiomyopathy within 5 months of delivery, 2) absence of an identifiable cause of cardiomyopathy, 3) absence of recognizable heart disease before the last month of pregnancy, and 4) left ventricular systolic dysfunction with left ventricular ejection fraction (LVEF) of $<45 \%(1,4)$. Furthermore, histological investigation of the endomyocardial biopsy revealed myocyte degeneration and interstitial fibrosis, which may support the diagnosis of PPCM.

This PPCM patient showed recovery of ventricular function within 4 months after onset of syncope ( 5 months postpartum). Elkayam et al reported that recovery of LVEF $(>50 \%)$ was observed in $54 \%$ of patients, and occurred within 6 months postpartum in most patients, which is distinct from other forms of non-ischemic cardiomyopathy. An improvement in LVEF at the last follow-up is significantly larger in women with an LVEF of $>30 \%$ at time of diagnosis (5). For this reason, the present patient whose initial LVEF was $43.6 \%$ would be considered to be in a favorable position for spontaneous recovery. Similar to the medical management of patients with other forms of cardiomyopathy, ACE inhibitors and/or beta-blockers are commonly used for PPCM. Apart from the hemodynamic benefits of these classes of drugs, they may have the additional benefit of decreasing an overactive immune system, which plays a role in the basic pathophysiology of PPCM (6). Thus, the treatment of this patient with enalapril, an ACE inhibitor, from the first day after admission may have facilitated the improvement in PPCM.

The KCNH2 gene encodes the $\alpha$-subunit of the voltagegated potassium ion channel (Kv11.1, also called hERG), which plays a crucial role in ventricular repolarization. The role of $\mathrm{KCNH} 2$ is of particular pathophysiological importance, because mutations in this gene have been linked to the inherited type 2 long QT syndrome (LQT2) (3). We identified a novel $\mathrm{KCNH} 2$ gene mutation in the present patient and in 2 of her family members. This mutation was a 4-bp insertion leading to a C-terminal truncation of the hERG channel. Choe et al reported that functional assays 
with $3 \mathrm{C}$-terminal truncating mutations in $\mathrm{KCNH} 2$ identified in the LQT2 families suggests an impaired ability of Cterminally truncated hERG protein to regulate channel activity in response to $\beta$-adrenergic stimulation (7). Based on their findings, we suspect that this novel mutation producing a truncated hERG protein at amino acid 920 should have a similar effect, although the functional effect of this mutation has not yet been clarified in vitro. Mild electrolyte abnormalities and cardiac failure due to PPCM led to marked QTc prolongation, and both the patient's QTc after recovery and those of her family members carrying this mutation were slightly prolonged (within border range), a finding that could be partly explained by this KCNH2 mutation. However, nevertheless, the patient's first delivery was uneventful, and her mother and sister were clinically unaffected by this mutation. Based on these facts, this mutation does not appear to have a strong impact on the phenotype of LQTS.

Women with LQT2, even without PPCM, are at increased risk for cardiac events during the postpartum period (8). However, it is difficult to predict cardiac events when such genetic mutations are not identified. Therefore, we recommend that the QT interval be measured in every pregnant woman, even those without preexisting cardiac diseases or a history of syncope to help prevent cardiac events, i.e., by avoiding hypokalemia and hypomagnesemia. In conclusion, we encountered a rare case of PPCM with recurrent syncope due to TdP. In this patient, inherited LQTS with $\mathrm{KCNH} 2$ mutation was unmasked via exposure to electrolyte disturbance and structural cardiac failure due to PPCM.

The authors state that they have no Conflict of Interest (COI).

\section{References}

1. Pearson GD, Veille JC, Rahimtoola S, et al. Peripartum cardiomyopathy: National Heart, Lung, and Blood Institute and Office of Rare Diseases (National Institutes of Health) workshop recommendations and review. JAMA 283: 1183-1188, 2000.

2. Gemici G, Tezcan H, Fak AS, Oktay A. Peripartum cardiomyopathy presenting with repetitive monomorphic ventricular tachycardia. Pacing Clin Electrophysiol 27: 557-558, 2004.

3. Roden DM, Viswanathan PC. Genetics of acquired long QT syndrome. J Clin Invest 115: 2025-2032, 2005.

4. Demakis JG, Rahimtoola SH. Peripartum cardiomyopathy. Circulation 44: 964-968, 1971.

5. Elkayam U, Akhter MW, Singh H, et al. Pregnancy-associated cardiomyopathy: clinical characteristics and a comparison between early and late presentation. Circulation 111: 2050-2055, 2005.

6. Godsel LM, Leon JS, Engman DM. Angiotensin converting enzyme inhibitors and angiotensin II receptor antagonists in experimental myocarditis. Curr Pharm Des 9: 723-735, 2003.

7. Choe CU, Schulze-Bahr E, Neu A, et al. C-terminal HERG (LQT 2) mutations disrupt IKr channel regulation through 14-3-3epsilon. Hum Mol Genet 15: 2888-2902, 2006.

8. Seth R, Moss AJ, McNitt S, et al. Long QT syndrome and pregnancy. J Am Coll Cardiol 49: 1092-1098, 2007. (C) 2012 The Japanese Society of Internal Medicine
http://www.naika.or.jp/imindex.html 